\title{
Design of intelligent integrated monitoring system under multistation fusion platform
}

\author{
Lianteng Shen ${ }^{1,}$, Ling $\mathrm{Li}^{1}$, Zhe $\mathrm{Li}^{1}$, Xin Zhang ${ }^{1}$, and Junjie $\mathrm{Ma}^{2}$ \\ ${ }^{1}$ China Electric Power Research Institute, 100192, Beijing, China \\ ${ }^{2}$ School of Electronics and Information Engineering, Anhui University, 230601, Hefei, China
}

\begin{abstract}
With the continuous advancement of the national energy strategy of China, constructing multistation fusion platform (MSFP) of substations, energy storage stations, and data center stations by taking advantage of the remaining resources of substations is a development trend. The monitoring of equipment operation and environmental parameters is a powerful guarantee for the stable and reliable operation of MSFP. Thus, this study developed an intelligent integrated monitoring system construction method that consists of state perception, information fusion, and decision and control layers. First, the state of equipment and environmental parameters were monitored efficiently through built-in status signals and multisensing techniques. Then, the multilevel transmission network, composite data structure, and data interface are integrated to achieve the collection of multisite information. Moreover, the device object model and station-level digital model were designed. Finally, the abnormal fault diagnosis of equipment, data fusion regulation, and coordinated optimization control were completed by using knowledge graph, data optimization and neural network modeling, and hierarchical analysis of multiple application scenarios in MSFP. The intelligent monitoring system can monitor and control the equipment, operation, and environment in the MSFP efficiently, thereby providing technical support for the new development of China's electric power industry.
\end{abstract}

\section{Introduction}

Driven by the "Energy Internet" of the State Grid Corporation of China, the integration of energy flow, data flow, and service flow is an important development trend. The use of the remaining substation resources to achieve the "multistation integration" of substations, energy storage stations, and data center stations is the core of the future business in state grid [1]. Multistation fusion platform (MSFP) can share hardware resources; accelerate the construction process; save the costs of lines planning, cables laying and cable channels; promote the quality and stability of services of various types of stations; and increase investment income [2-4]. To monitor the equipment status and operation of each station in real time, generating control strategies and conducting control actions based on the monitoring data is a key issue for the stable, high-quality, and reliable operation of the MSFP.

\footnotetext{
*Corresponding author: apcomm_2010@163.com
} 
At present, experts and scholars have explored the integrated construction and operation of substations, energy storage stations, and data center stations. Cui et al. designed a fusion architecture based on a full DC bus; this architecture saved some links of power conversion required by the $\mathrm{AC}$ microgrid and provided a feasible way of new energy consumption, economical operation, and reliable operation of the system [5]. Zhang et al. discussed the connotations and characteristics of multistation fusion and the design and feasibility of the operation model, service system, and business model [6]. Li et al. considered the individual demands of different loads, and the solution method is proposed to minimize multidimension load distance cost based on Voronoi diagram theory. The case study simulates the application of this model and its comparison with traditional substation planning, thereby verifying the feasibility of the proposed model [7]. Meng Chao et al. designed a fusion station operation architecture that covered the data center and energy storage power station and constructed a load model of data center and a mathematical model of energy storage station. Moreover, a collaborative optimization and data migration scheduling model of an integrated station was established [8]. However, problems about independent development, independent operation, and decentralized management of MSFP, including great heterogeneity in terms of implementation, environment and platform, and difficulties in data exchange, application connectivity, and functional linkage, were developed by the monitoring system for various types of stations [9-12]. Therefore, an intelligent integrated monitoring platform is necessary for MSFP to monitor the status of the station efficiently, gather the monitoring data of various types of stations quickly, and mine heterogeneous data information simultaneously.

\section{Design of intelligent integrated monitoring system for MSFP}

In the MSFP, the integrated monitoring system needs to supervise and regulate the operation status of substations, energy storage stations, and data center stations timely and reasonably. The monitoring system can be divided into a state perception layer, an information fusion layer, and a decision and control layer (Figure 1). The state perception layer obtains the operating parameters of various types of stations. Substations need to perceive power quality information, such as the quantity of electricity as voltage and power, the status of auxiliary devices as press plates, circuit breakers, and isolating switches, as well as information on environmental parameters in the stations [13]. Energy storage station is necessary to monitor battery management systems, energy storage converters, water leakage, and fires [14]. The data center stations need to sense ambient temperature and humidity, water leakage, fire, and access control [15]. However, the lighting and air-conditioning status in the station are related to various stations. Consequently, the overall status of the device is fully perceived by selecting the appropriate sensor unit and optimizing the layout while sharing hardware resources to obtain high performance. The information fusion layer completes the collection and sorts and store the aforementioned multiparameter heterogeneous monitoring data. It also involves the design of the network transmission system, the conversion of heterogeneous data, the placement of storage equipment, and the interaction with the decision center. Multilevel jump, local backup, and cross-site fusion are used to ensure the accurate, timely, and reasonable data transmission. The decision and control layer analyzes the integrated data, generates coordinated optimization decision, layouts the control equipment, and implements control. In this process, the integration of data center stations, energy storage stations, and substations should be considered for grid-connected, off-grid, demand side response, network load interaction, grid auxiliary services, and other unused application scenarios. Analytic hierarchy process and the establishment of a knowledge base for specific scenarios are adopted to complete the coordination and intelligent control of the three main bodies and ensure the stable, reliable, and efficient operation of the MSFP. 


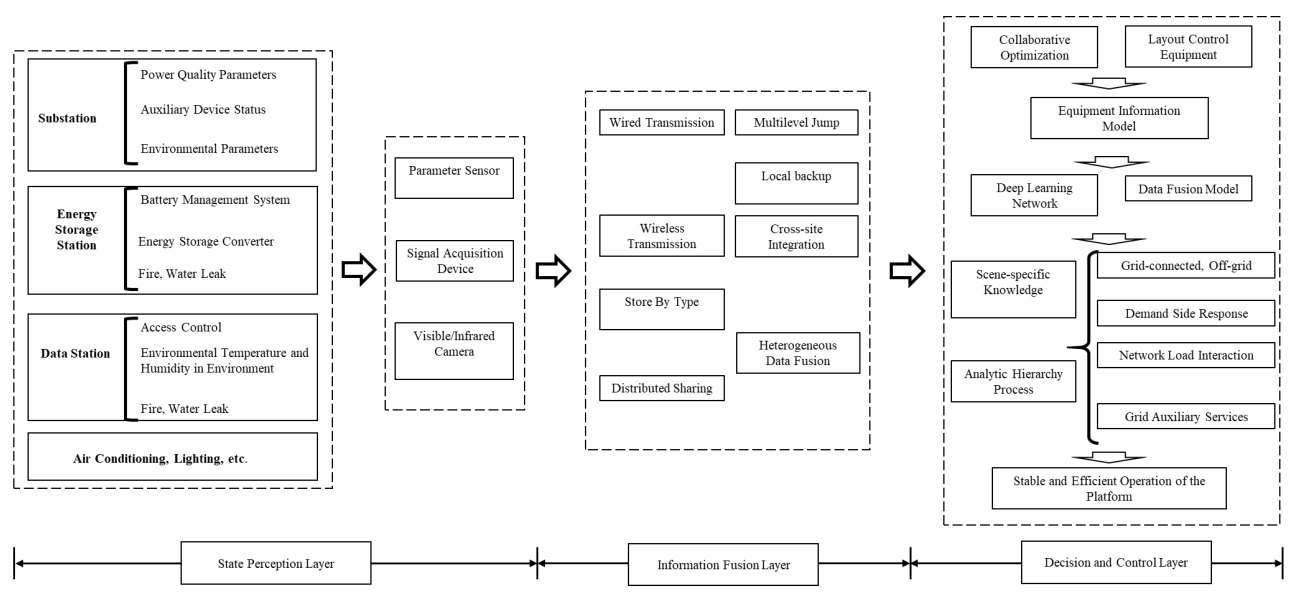

Fig. 1. Structure of the integrated monitoring system.

\section{Hierarchical realization of intelligent integrated monitoring system for MSFP}

\subsection{State perception layer}

Monitoring the operation status of substations, energy storage stations, and data center stations will provide an important reassurance for the stable and efficient operation of the MSFP. First, a state perception layer is established. Second, the state perception layer should be oriented to the monitoring requirements of equipment and state in substations, energy storage stations, and data center stations. Third, the integration of multiple technical means and reasonable layout is considered to obtain comprehensive and accurate state information.

Substation monitoring is complicated and important. First, power quality data, such as voltage, current, power, power factor, frequency, harmonics, unbalance rate, as well as auxiliary device information, including circuit breaker position, protection plate status, isolating switch, and transformer tap alarm must be monitored. In addition, environmental parameters, including temperature (air, water and cables), humidity, and light, should also be monitored. The energy storage stations focus on the operating status of battery management systems, energy storage converters and water leakage, fire, and other situations. The internal operation of the data center stations is controlled by the internal operation and maintenance system. At the site integration level, attention should be paid to the environmental temperature and humidity, water leakage, fire, and access control parameters of the data center station. The air conditioning, lighting, and access control information in the entire fusion platform need to be sensed simultaneously.

The monitoring of power quality can be achieved by monitoring the running condition of power supply lines. The low-voltage same-frequency signal is obtained for the high-voltage input end of the power supply line through the AC transformer. Subsequently, the lowvoltage signal is connected to the analog-to-digital (AD) converter sampling end of the microcontroller system, and the time domain signal is obtained by the sampler. Thereafter, the voltage, current, power, power factor, and other power parameters of the low-frequency signal are calculated, and the actual power quality signal in the actual substation is inverted by the principle of transformer coupling. Based on the aforementioned idea, we designed a signal acquisition device based on STM32 microcontroller (Figure 2). The main control chip of this device is STM32F407, which adopts STMicroelectronics $90 \mathrm{~nm}$ process and ART 
accelerator, has dynamic power adjustment function, and can achieve a current consumption that is as low as $238 \mu \mathrm{A} / \mathrm{MHz}$ (@ $168 \mathrm{MHz}$ ) in run mode or executing from Flash memory. DSP instructions and floating-point units expand the application range of the product. For example, up to 15 communication interfaces, including 6 USART with speed of up to 11.25 $\mathrm{Mb} / \mathrm{s}, 3 \mathrm{SPI}$ with speed of up to $45 \mathrm{Mb} / \mathrm{s}, 3 \mathrm{I}^{2} \mathrm{C}, 2$ CAN And $1 \mathrm{SDIO}, 3 \mathrm{ADC}$ of 12-bit with a speed of 2.4 MSPS or 7.2 MSPS, and multiple high-speed timers. Thus, expanding storage capacity and accelerating hardware are easy.

The status of the battery management system and the energy storage converter in the energy storage stations, as well as the air conditioner in the environment and the access control, can also be collected directly by the aforementioned status collection device. The temperature, humidity, light, and other parameters in the environmental parameters are performed using the Aosong-AM2105A dust-proof temperature and humidity sensor and Opt3001 light sensor, and the collected values are also inputted to the signal collection device for collection. Certainly, the perception of water leakage and fire information in the environment should be integrated with multiple sensor technologies to obtain earlier and more accurate detection. Water leakage requires the arrangement of a humidity sensor (SAISITE-SHT30) and a digital image camera (Hikvision DS-2DC2402IW-D3/W) at key locations. The fire detection can be achieved by combining a smoke sensor (Jingyan Electronics MQ-2) and infrared camera (Hikvision 3325D-I). The data of the humidity sensor and the smoke sensor can be connected to the self-designed signal acquisition device, and the camera has its own network transmission module that can accomplish the data mutual transmission. The auxiliary equipment in the substation, such as the position of the circuit breaker, the status of the protection plate, and the connector of the transformer, are also visually detectable, and the network digital camera can be directly used for status collection.

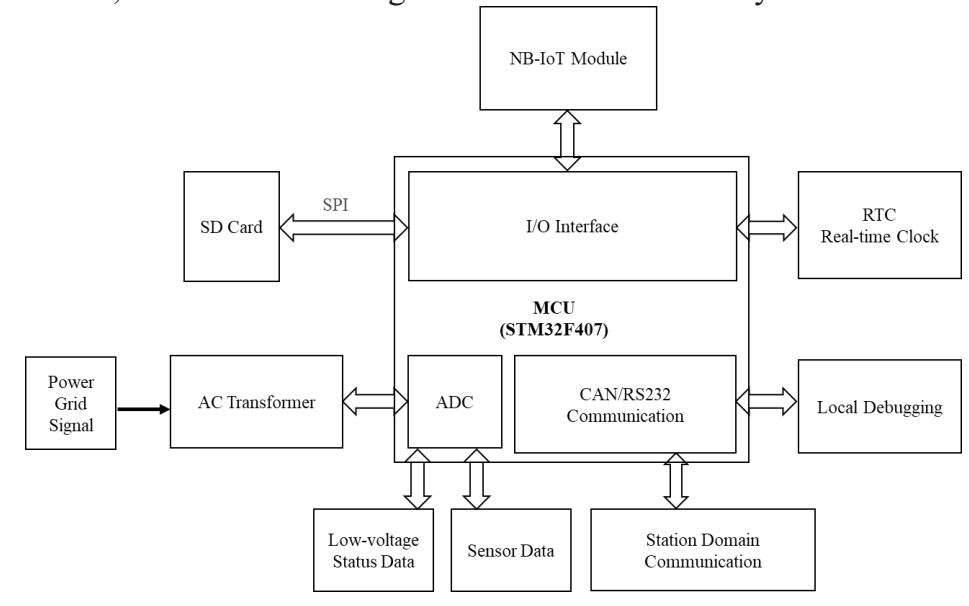

Fig. 2. Signal acquisition device based on STM32.

\subsection{Information fusion layer}

After completing the hardware layout of the state perception layer under MSFP, the information fusion layer of the intelligent integrated monitoring needs to collect, process, and store all the state monitoring data preliminary. The standardized definition of communication content, communication frequency, and communication protocol is provided by establishing standardized communication channels to ensure the safety and convenience of data collection and transmission in the MSFP. Then, the data storage of the multistations in the data center stations and the collection and comprehensive integration of professional data in the data 
center stations are conducted to meet the needs of local and remote data application and maintenance. Specifically, the information convergence layer includes the communication network architecture and the design of integrated data structure. The communication network performs wired and wireless transmissions and is realized by multilevel jump.

The power quality in the substation, the battery management system in the energy storage station, the energy storage converter, the access control system of the data center stations, the lighting and air conditioning and other status monitoring data, as well as the temperature, humidity, light, and smoke sensors in the environmental parameters, can be transmitted to the signal acquisition device in a single station through the wired CAN bus. Then, the subdata collection point, external SD storage card, and the data storage unit are constructed. Considering that the narrow band Internet of Things (NB-IoT) has the characteristics of wide coverage, massive connections, low cost, and low power consumption, the NB-IoT based on the BC35-G module is adopted to connect the network server in the data center stations. Monitoring data from cameras and infrared cameras can be transmitted through the local wireless network in MSFP, and the wireless data collection nodes are set up at the data center stations. Electric energy intelligent monitoring devices and cameras should be equipped with network hard disks, and the kind of data is critical and has a large amount of data. Therefore, the local backups should be created to achieve the backtracking of large amounts of data.

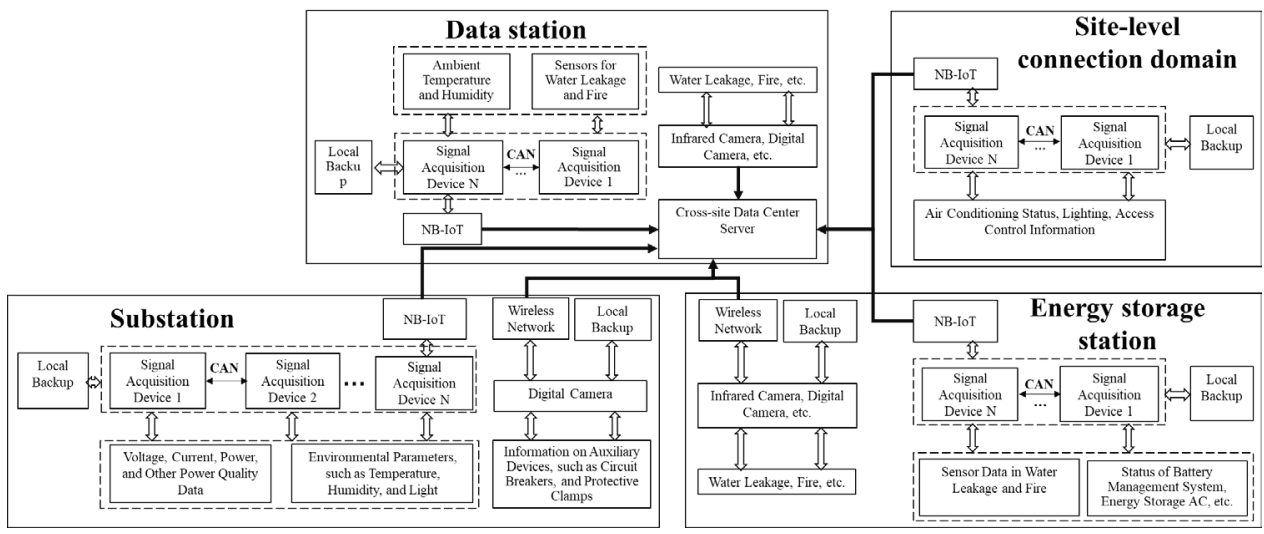

Fig. 3. Monitoring network architecture under MSFP.

Monitoring data from different sources is stored in different formats. The storage of image data adopts BMP bitmap format, and the data storage method uses cube matrix storage. The equipment condition monitoring data and environmental sensor data in the three stations are in small size and low in receiving frequency. These data can be directly stored in text format, even if the storage format is the same, the data format varies. The integration of the abovementioned formats will improve the efficiency of information fusion while reducing costs. The fusion of heterogeneous data should follow the following rules [16]:

(1) The docking interface shall be compatible with the current basic equipment of the state grid standard of China and must expand the capacity of devices in the future ubiquitous IoT by building an interface platform to provide unified interface open docking functions for the outside world to achieve a high cohesive interface platform specification standard.

(2) The priority of data sources should be sorted according to the operating status of the core equipment of each type of station, the status of auxiliary equipment in the station, the hazardous parameters in the environmental parameters, and other environmental parameters to prevent collisions among data transmissions.

(3) Data time must be synchronized. Moreover, time stamps should be set to ensure the consistency of data running across multiple sites to avoid the data confusion in time. 
Meanwhile, in the processing framework based on the Hadoop distributed data, multiple distributed databases are embedded in the shared platform in a modular form to achieve rapid segmentation and merging. Serial parallel data processing flow flexibly completes the storage and calculation of fragmentary data, and the data extension of the new station can be completed by embedding the new database module. The data between multiple types of sites can also be used as one another's image backup site. When the data of a site are lost, the original data can be recovered from the image of other sites, thereby enhancing the robustness of data center processing capabilities and completes the monitoring data in the site. These data can be consulted and called in time to support global business data.

\subsection{Decision and control layer}

The decision and control layer has a series of functions of big data mining and data modeling processing, such as real-time warning, data analysis, and data processing (Figure 4). The decision and control layer analyzes the information characteristics and filters the data of each object involved in the multistation integrated power station according to the requirements and characteristics of the MSFP. Unified modeling language (UML) is used to organize the information data of each object, and the common information model (CIM) of multistation fusion devices is established. According to the obtained data information, the knowledge graph method was adopted to analyze the poor operating status of the equipment under MSFP and to guide the elimination of equipment defects, providing the equipment abnormal location, fault diagnosis, real-time alarm and historical query. Multisite integrated display is completed in the same knowledge map site, and the aggregate display of the site and the integrated management of MSFP monitoring are implemented from the perspective of operation and maintenance.

Subsequently, to establish digital simulation models for substations, energy storage stations, and data center stations, the state of the power grid is simulated under various conditions of reactive and active power. Then, the back feedback neural network (BPNN) and logic analysis algorithm are used to carry out the optimal control strategies of the power grid. Moreover, the data optimization of the control strategy is determined by analyzing the data required by the control strategy, the data acquisition mode, and the data response delay. After analyzing the past MSFP data with the help of recurrent convolutional network (RNN) and statistical data, a data fusion control model is established to provide the data support function of data-supported intelligent scheduling for the "three stations in one" intelligent scheduling and achieve the supporting ability of cross-site resource scheduling and comprehensive scheduling.

According to the characteristics of the established multisite fusion model, the decision and control layer uses the analytic hierarchy process to obtain coordinated and optimized control strategies in different application scenarios, such as grid-connected, off-grid, demand-side response, grid-load interaction, and grid auxiliary services for data center and energy storage station integration with the grid. Then, a multistation fusion station level energy optimal scheduling control strategy based on dynamic unified time domain model, including local adaptive optimal economic operation control strategy, data center for power supply security and reliability, operation control method and strategy for multitype energy storage and power grid, the operation control method of optical storage and other aggregated resources involved in the demand side response and auxiliary service of power grid, is established. The reactive power is intelligently distributed by utilizing the reactive power regulation capability of compensation capacitor, energy storage, and distributed power supply comprehensively to achieve accurate compensation and minimize the operation frequency. The intelligent control of multiload active power and optimal operation of the system are performed by taking advantage of the efficient operation state of the transformer 
in the station, the level of system voltage frequency, the working state of the distributed power supply, and the load state and the information of working period. The influence of the grid side fault or system disturbance on the running state of energy storage are explored for joint control. Moreover, the impact of grid power flow on the protection and automatic control functions is analyzed for the optimal energy storage power generation mode.
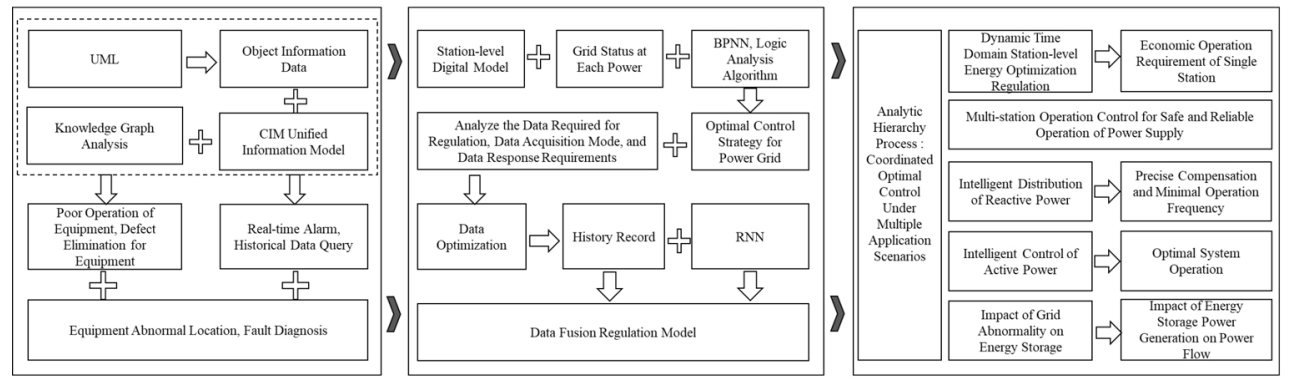

Fig. 4. Control strategies of the decision control layer of the monitoring system.

\section{Conclusion}

Under MSFP of substations, energy storage stations, and data center stations, this work proposed an intelligent integrated monitoring system that consists of a state-aware layer, an information fusion layer, and a decision and control layer. The built-in status signal, multisensor technology equipment status, and environmental parameters are monitored effectively by combining multilevel transmission network, composite data structure, and integrated data interface to complete the multistation data information collection. Moreover, the accurate control and scheduling of the three-station operation is achieved by using the device model, station level digital model, and multistation interactive association relation. The monitoring system is expected to provide system support for the stable operation and efficient work of the MSFP. We believe that the intelligent monitoring system would provide a new boost for the development of the power grid.

This study is supported by Key Technology of "Three Stations in One" of Substation, Charge and Discharge (Energy Storage) Station and Data Center Station.

\section{References}

1. Y. Zhang, C. Zhang, Y. Zou, J. Chen, L. Sun, Z. Yang, C. Xiao, Conf. AEECA, 576581(2020)

2. B. Wang, Y. Zhang, M. Liu, X. Mi, Electric Power Information and Communication Technology, 17, 41-45 (2019)

3. W. Xu, H. Cheng, Z. Bai, C. Miao, F. Sun, Power Supply, 36, 84-91 (2019)

4. C. Duojie, L. Zhang, X. Wu, Q. Guo, Qinghai Electric Power, 38, 24-26 (2019)

5. H. Cui, B. Yang, Y. Tang, Y. Li, G. Chen, Power Supply and Consumption, 37,1620(2020)

6. Y. Zhang, B. Wang, R, Li, X. Mi, Power Supply, 36,62-66 (2019)

7. X. Li, J. Wang, J. Ding, T. Shan, Y. Cheng, Power System and Clean Energy, 36,44-54 (2020)

8. C. Meng, W. Liu, Q. Yang, Y. Zhao, Y. Guo, Eng. Sci. Technol, 52,49-55 (2020)

9. Y. Tian, Z. Pang, W. Wang, L. Liu, D. Wang, Conf. ITNEC, 1613-1617 (2017) 
10. K. Wei, Y. Xiang-wu, L. He-ming, Z. Li-xia, Conf. IPEC, 420-424 (2006)

11. M. T. Lawder, B. Suthar, P. W. Northrop, S. De, C. M. Hoff, O. Leitermann, V. R. Subramanian, Proc IEEE Inst Electr Electron Eng, 102, 1014-1030 (2014)

12. Z. Fang, S. Zhou, H. Ge, Commun. Technol, 42,129-131(2009)

13. D. Gao, L. Wang, J. Comput. Methods Sci. Eng, 19, 219-226 (2019)

14. Y. Li, X. Li, X. Jia, R. Ma, D. Hui, Conf. IICSPI, 765-769 (2018)

15. Z. You, W. Mao, Q. Wu, Y. Guo, Conf. CICED, 1256-1262 (2018)

16. X. Yuan, W. Liu, C. Sun, N. Yang, Electric Power Information Technology, 18,4651(2020) 